\title{
Spontaneous pneumomediastinum in a young
}

\section{woman}

\begin{abstract}
Spontaneous pneumomediastinum is a rare clinical entity, defined as the presence of free air in the mediastinal structures without any apparent cause. However, physicians often fail to consider spontaneous pneumomediastinum in the differential diagnosis because of its spontaneous healing and rare occurrence. A previously healthy 28 -yearold woman suddenly developed left-sided chest pain while eating lunch, without any cough or vomiting. She presented for treatment because of persistent chest pain and anxiety. Her vital signs were stable on admission. She had not taken any medications recently, nor reported a habit of cigarette smoking. Physical examination revealed no aerodermectasia or abnormal chest sounds, including crunching heart sounds. The electrocardiogram was normal. Chest radiographs showed air dissecting the left second arc protrusion and in the superior mediastinum. Computed tomography of the chest revealed air in the mediastinum, as well as aerodermectasia not detected in the radiographs, without pneumothorax or oesophageal rupture. An esophagogram demonstrated no leakage in the mediastinum or pleural space. These findings confirmed a diagnosis of spontaneous pneumomediastinum. The patient's chest pain improved gradually, and spontaneously resolved completely within 1 week with conservative management.
\end{abstract}

Volume 2 Issue 3 - 2018

\author{
Takayuki Yamada,' Susumu Ohwada² and \\ Takashi Ogino ${ }^{3}$ \\ 'Asunaro Clinic, Takasaki city, Japan \\ ${ }^{2}$ MSS Ohta Central General Hospital, Japan \\ ${ }^{3}$ Honjo Waseda Clinic, Japan
}

Correspondence: Takayuki Yamada, Asunaro Clinic, Takasaki city, Gunma Pref, Japan, Tel +8I-27-372-8060,

Email asunaro.clinic.takasaki@gmail.com

Received: April 21, 2018 | Published: May 08, 2018

Keywords: spontaneous pneumomediastinum, diagnosis, mediastinum, pleural space, esophagogram, superior mediastinum, clinical entity, eating lunch, chest radiography, abnormal chest, aerodermectasia

Abbreviations: SPM, spontaneous pneumomediastinum; CT, computed tomography; ECG, electrocardiogram

\section{Case presentation}

Spontaneous pneumomediastinum (SPM) is a rare clinical entity defined as the presence of free air in the mediastinal structures without any apparent cause. The incidence of this disease has been reported to range from 1 in 30000 to 1 in $44500 .^{1,2}$ However, physicians often fail to consider SPM in the differential diagnosis because of its spontaneous healing and rare occurrence.

A previously healthy 28-year-old woman suddenly developed left chest pain while eating lunch, with no cough or vomiting. She visited my office because of persistent chest pain and anxiety. Her vital signs were stable on admission. She had not taken any medications recently, nor reported a habit of cigarette smoking. Physical examination revealed no aerodermectasia or abnormal chest sounds, including crunching heart sounds. The electrocardiogram (ECG) was normal. Chest radiographs showed air dissecting the left second arc protrusion Figure 1, black arrow and in the superior mediastinum Figure 1, black arrow head. Computed tomography (CT) of the chest also revealed air in the mediastinum Figure 2, white arrow, as well as aerodermectasia Figure 2, arrow head not detected in the radiographs, without pneumothorax or oesophageal rupture. An oesophagogram demonstrated no leakage in the mediastinum or pleural space. These findings confirmed a diagnosis of SPM. The patient's chest pain improved gradually, and spontaneously resolved completely within 1 week with conservative management.

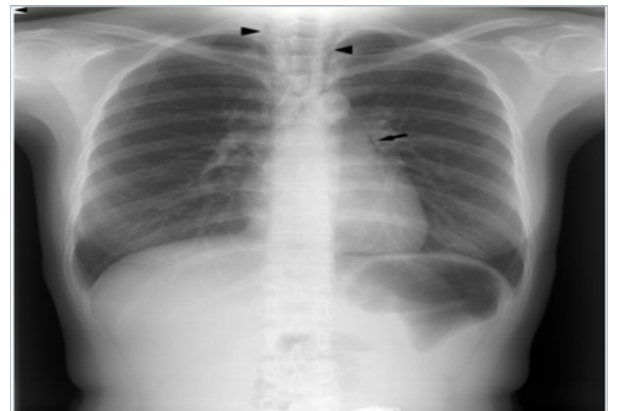

Figure I A chest radiograph showing intramediastinal air.

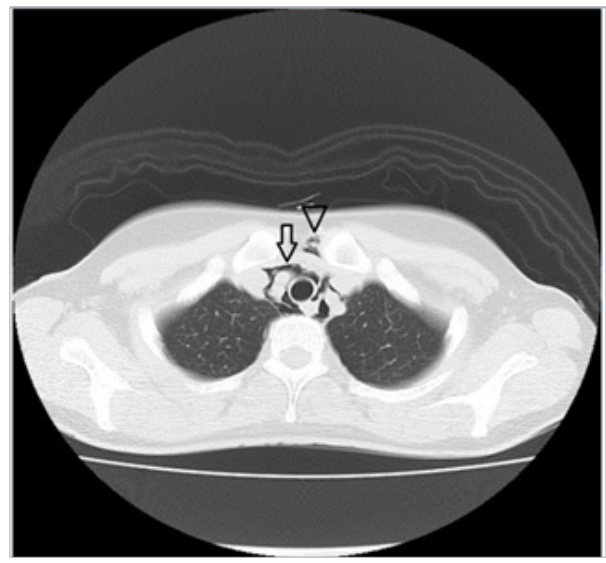

Figure 2 A chest CT showing intramediastinal air (white arrow) and aerodermectasia (white arrow head) without pneumothorax. 


\section{Discussion}

Chest radiography confirmed the disappearance of intramediastinal air. The incidence of SPM may be underestimated because it heals spontaneously and the presence of intramediastinal air can be easily missed in a primary care setting. In addition, the patient may not seek medical assessment. Among the many causes of sudden-onset chest pain, the main differential diagnoses include oesophageal perforation, acute coronary syndrome, pericarditis, pneumothorax, musculoskeletal disorders, and pulmonary embolism. Secondary pneumomediastinum caused by oesophageal perforation, which results in a mediastinal abscess and is associated with a poor prognosis, should be considered in the differential diagnosis. It can be a consequence of chest bruising, a severe cough, or the Valsalva manoeuvre, none of which occurred in the patient described here. CT and contrast oesophagography should be performed for SPM diagnosis. SPM occurs most frequently in young males and is rare in children. It typically develops after alveolar rupture and air penetration into the pulmonary interstitium, followed by air penetration towards the hila and into the mediastinum. Alveolar ruptures may be caused by various pathological and physiological processes, most frequently by bronchial asthma. Clinical diagnosis of an alveolar rupture is based on a triad of symptoms including chest pain, dyspnoea, and aerodermectasia. ${ }^{3}$ Complications of spontaneous pneumomediastinum are intramediastinal abscesses and tension pneumothorax, which occur rarely but may be fatal if left untreated. A patient with a confirmed diagnosis of SPM should be followed closely by a physician for several days.

\section{Conclusion}

When examining young patients with sudden-onset chest pain, physicians should consider SPM and carefully confirm the presence or absence of intramediastinal air. SPM occurs rarely; however, severe complications, including intramediastinal abscess formation and tension pneumothorax, may result in a poor prognosis. The patient should remain under a physician's close supervision until recovery.

\section{Acknowledgements}

None.

\section{Conflict of interest}

The authors have stated explicitly that there are no conflicts of interest in connection with this article.

\section{References}

1. NewcombeAE, Clarke CP, ClarkeP. Spontaneous pneumomediastinum: a benign curiosity or a significant problem? Chest. 2005;128(5):32983302 .

2. Macia I, Moya J, Ramos R, et al. Spontaneous pneumomediastinum: 41 cases. Eur J Cardiothorac Surg. 2007;31(6):1110-1114.

3. Dajer Fadel WL, Argüero Sánchez R, Ibarra Pérez C, et al. Systematic review of spontaneous pneumomediastinum: a survey of 22 years' data. Asian Cardiovasc Thorac Ann. 2014;22(8):997-1002. 